\title{
Direct Growth of Graphene on Silicon by Metal-Free Chemical Vapor Deposition
}

\author{
Lixuan Tai ${ }^{1,3} \cdot$ Daming Zhu ${ }^{1} \cdot$ Xing Liu $^{1,2} \cdot$ Tieying Yang ${ }^{1} \cdot$ Lei Wang ${ }^{1} \cdot$ Rui Wang $^{1} \cdot$ Sheng Jiang $^{1}$. \\ Zhenhua Chen ${ }^{1} \cdot$ Zhongmin $\mathrm{Xu}^{1} \cdot$ Xiaolong $\mathrm{Li}^{1}$
}

Received: 6 September 2017/Accepted: 6 November 2017/Published online: 8 December 2017

(C) The Author(s) 2017. This article is an open access publication

\section{Highlights}

- Graphene was successfully grown on single-crystal silicon substrates using metal-free, ambient-pressure chemical vapor deposition.

- Atomically flat monolayer or bilayer graphene domains, concave bilayer graphene domains, and bulging few-layer graphene domains can be produced by controlling the growth temperature.

- In-plane propagation, edge-propagation, and core-propagation processes are proposed to evaluate the sequentially changing graphene domains.

\begin{abstract}
The metal-free synthesis of graphene on singlecrystal silicon substrates, the most common commercial semiconductor, is of paramount significance for many technological applications. In this work, we report the growth of graphene directly on an upside-down placed, single-crystal silicon substrate using metal-free, ambientpressure chemical vapor deposition. By controlling the
\end{abstract}

Electronic supplementary material The online version of this article (https://doi.org/10.1007/s40820-017-0173-1) contains supplementary material, which is available to authorized users.

Lixuan Tai, Daming Zhu, and Xing Liu contributed equally to this work.

Xiaolong Li

lixiaolong@sinap.ac.cn

Daming Zhu

zhudaming@sinap.ac.cn

1 Shanghai Synchrotron Radiation Facility, Shanghai Institute of Applied Physics, Chinese Academy of Sciences, Shanghai 201204, People's Republic of China

2 University of Chinese Academy of Sciences, Beijing 100049, People's Republic of China

3 Department of Electronic Engineering, Tsinghua University, Beijing 100084, People's Republic of China

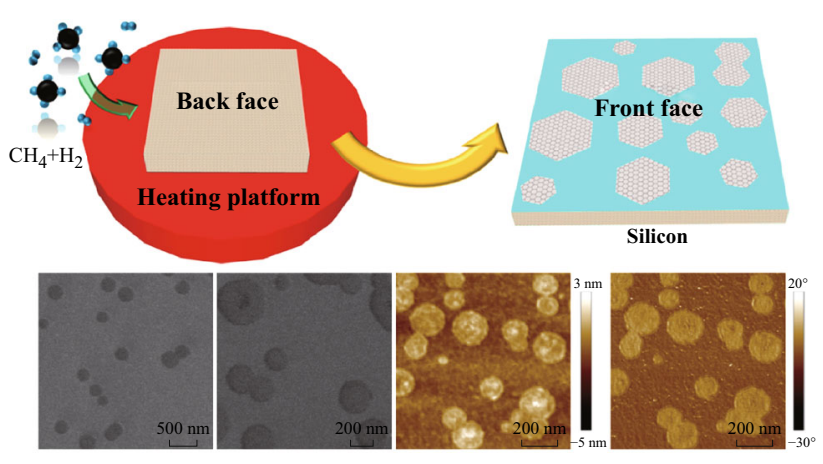

growth temperature, in-plane propagation, edge-propagation, and core-propagation, the process of graphene growth on silicon can be identified. This process produces atomically flat monolayer or bilayer graphene domains, concave bilayer graphene domains, and bulging few-layer graphene domains. This work would be a significant step toward the synthesis of large-area and layer-controlled, high-quality graphene on single-crystal silicon substrates.

Keywords Graphene - Silicon · Metal-free CVD · Domain growth 


\section{Introduction}

Graphene is a novel two-dimensional material with remarkable mechanical and electrical properties, such as high carrier-mobility and outstanding mechanical flexibility [1]. Since the first graphene samples were produced via mechanical exfoliation of bulk graphite [2], various synthetic methodologies have been developed for graphene preparation [3-6]. Chemical vapor deposition (CVD) is one of the most efficient methods for the synthesis of large-area graphene films with high crystallinity and large grain size on metal substrates (e.g., copper, nickel) [4, 7]. However, in this conventional method, graphene grown on a metal surface must be transferred to the desired substrate (e.g., dielectrics, semiconductor) for applications. While waferscale transfer is possible, this requires complicated and costly transfer procedures, which tend to induce mechanical damage as well as chemical contamination [8]. In other words, it is highly desirable to produce high-quality graphene films on a wide range of substrates directly through CVD without a metallic catalyst.

For the past few years, interest in the direct growth of graphene on semiconductor substrates is increasing $[9,10]$ due to the unique properties of graphene-semiconductor interfaces, which result in synergetic effects in hybrid devices [11-13]. In particular, silicon plays an important role in the electronic and photoelectronic industries, and the development of hybrid graphene-Si structures/devices may offer a seamless integration of graphene into current microelectronics technology. In fact, graphene-silicon heterostructures have found their way into various applications including Schottky diodes [14], tunable ultrasensitive photodetection [15], and solar cells [16], which would present many more interface properties with the direct growth of graphene on silicon. So far, much research has been dedicated to the direct synthesis of graphene on $\mathrm{SiC}$ [17], $\mathrm{SiO}_{2}$ [18-22], quartz [23, 24], $\mathrm{Al}_{2} \mathrm{O}_{3}$ [25, 26], $\mathrm{Si}_{3} \mathrm{~N}_{4}$ [27], $\mathrm{SrTiO}_{3}$ [28], Ge [9, 10], and hexagonal boron nitride (h-BN) [29-31] substrates. Compared to the above substrates, single-crystal silicon has received relatively little attention for direct graphene growth via CVD. This is mainly due to its catalytically inert (compared to metal substrates) nature and the surface instability of singlecrystal silicon (compared to $\mathrm{SiO}_{2}$, quartz, $\mathrm{Al}_{2} \mathrm{O}_{3}, \mathrm{Si}_{3} \mathrm{~N}_{4}$, $\mathrm{h}-\mathrm{BN}$, etc.) during interaction with active hydrocarbon radicals at high temperature [4, 7, 18-31]. As a result, despite some studies of direct growth on single-crystal silicon substrates using metal-free CVD or solid-source molecular-beam epitaxy, only thick graphite carbon ( $\mathrm{SiC}$ may be accompanied in these processes) has so far been realized [32-34]. Overall, the quality of graphene directly grown on single-crystal silicon is still far from satisfactory.
In this paper, we demonstrate a novel method for the direct growth of graphene on an upside-down placed, single-crystal silicon substrate using metal-free, ambientpressure chemical vapor deposition (APCVD). Single graphene domains can be directly nucleated and grown on smooth, single-crystal silicon, regardless of crystal faces and doping levels. To the best of our knowledge, this work presents the first dedicated investigation of graphene growth on single-crystal silicon using metal-free CVD.

\section{Experimental}

\subsection{Metal-Free APCVD Growth of Graphene on Silicon Substrates}

Single-crystal silicon substrates $(500 \mu \mathrm{m}$ thick, (100), (111), and (110) face, $N$-type, $P$-type, intrinsic silicon, $R_{\mathrm{a}}<0.5 \mathrm{~nm}$ ) were purchased from Suzhou Crystal Silicon Electronic \& Technology Co. Ltd. A custom-made portable chamber, with a ceramic wafer as a heating platform, was used for the graphene growth. The temperature control was precisely calibrated with an error below $\pm 0.1{ }^{\circ} \mathrm{C}$. Before placement (upside-down) onto the heating platform, the single-crystal silicon substrates were pretreated by dipping into $15 \% \mathrm{HF}$ for $15 \mathrm{~min}$, and then cleaned by deionized water and dried by $N_{2}$ flow. The CVD chamber was evacuated and back-filled with argon (Ar) three times to create an inert environment. The singlecrystal silicon surface was then heated to the desired temperatures $\left(900-930^{\circ} \mathrm{C}\right)$ and stabilized for 10 min under $100 \mathrm{sccm} \mathrm{H}_{2}$ and $200 \mathrm{sccm}$ Ar to remove any organic residues and activate the growth sites. Typical graphene growth was performed with a gas mixture of $180 \mathrm{sccm} \mathrm{CH}$ and $10 \mathrm{sccm} \mathrm{H}_{2}$ for $1 \mathrm{~h}$. After that, the chamber was cooled down to room temperature in $1 \mathrm{~h}$ with $180 \mathrm{sccm}$ of $\mathrm{CH}_{4}$ and $10 \mathrm{sccm} \mathrm{H}_{2}$. As a comparison, the conventionally placed silicon substrates (faceup) were also used for graphene growth in the same processing conditions as that of the upside-down samples.

\subsection{Sample Characterization}

Atomic force microscopy (AFM) images were acquired with a Bruker Multimode 8 SPM with NanoScope version 8.15 software and a NanoScope V Controller using a tapping mode. The silicon tip with a nitride cantilever (SNL, $0.35 \mathrm{~N} \mathrm{~m}^{-1}$, Bruker) was treated with a plasma cleaner (Harrick Plasma, Plasma Cleaner PDC-32G) for 2 min prior to use. Raman spectra were recorded at room temperature using a Horiba Scientific LabRAM HR Evolution Raman spectrometer with a 473-nm wavelength laser. The laser spot size was about $2 \mu \mathrm{m}$, and the exposure time was 
30 s. High-resolution scanning electron microscopy (SEM) images were obtained by Model JSM-7600F (JEOL Ltd.). A monochromatic, Al $\mathrm{K} \alpha(h v=1436.6 \mathrm{eV}) \mathrm{X}$-ray source with a power of $36 \mathrm{~W}$ and a spot size of $40 \mu \mathrm{m}$ was used to collect spectra at a background pressure of $\sim 2 \times 10^{-9}$ torr. X-ray photoelectron spectroscopy (XPS, K-Alpha, Thermo Fisher Scientific Inc.) was used to characterize the samples. For the C 1s XPS spectrum, the binding energies were referenced to the $\mathrm{C} 1 \mathrm{~s}$ line at $284.8 \mathrm{eV}$.

\section{Results and Discussion}

Figure 1a shows a schematic diagram of the metal-free APCVD equipment used to grow graphene directly on single-crystal silicon. A custom-made, portable chamber with a ceramic wafer was used as the heating platform (Fig. S1). A detailed description of the chamber is also presented in our previous report [35]. The silicon substrate is mounted face down on the heating stage with a narrow gap between the $\mathrm{Si}$ and the heating stage. Unlike a commercial CVD chamber for graphene growth, the special design of the chamber used in this work remarkably increases the local concentration of hydrocarbon radicals and $\mathrm{C}$ ions, and enhances interaction with the silicon surface when the substrate is placed upside-down. Moreover, the confined hydrocarbon radicals and the increased collision frequency in the narrow gap further facilitate the nucleation of graphene [36]. This assumed mechanism is reasonable because the graphene was only observed on the upside-down surface. On the other hand, conventionally placed substrates cannot produce graphene or carbon nucleation at comparable temperatures.

Figure 1b shows a typical AFM image of a singlecrystal silicon (100) surface after graphene growth at $910{ }^{\circ} \mathrm{C}$. After exposure to the reaction gas mixture $\left(\mathrm{CH}_{4}: \mathrm{H}_{2}=180: 10 \mathrm{sccm}\right)$ for $1 \mathrm{~h}$, the silicon surface was covered with graphene domains. The thickness of the graphene domains was $\sim 0.7 \mathrm{~nm}$ (Fig. 1c), which is approximately equal to the thickness of a graphene monolayer [27, 28]. Some nearest-neighbor graphene domains can also link together and produce stacked graphene to form a bilayer with a thickness of $\sim 1.2 \mathrm{~nm}$ (Fig. 1b, c, line I). An XPS full scan of the as-grown sample proves that the growth of graphene on silicon is a complete, metal-free process without any metal contaminants (Fig. S2). Raman spectra were obtained for the single-crystal silicon surface after direct growth. Three prominent peaks were found and assigned at $1353 \mathrm{~cm}^{-1}$ (D band), $1592 \mathrm{~cm}^{-1}$ (G band), and $2710 \mathrm{~cm}^{-1}$ (2D band), confirming the formation of graphene (the prominent D peak should originate from the large number of domain boundaries and H-terminated graphene edges). The weak peak located at $\sim 1450 \mathrm{~cm}^{-1}$ can be attributed to the $\mathrm{C}-\mathrm{C}$ stretching and $\mathrm{C}-\mathrm{H}$ bending vibration $[37,38]$.

Figure $2 \mathrm{a}$, b displays the high-resolution SEM images of the graphene grains directly grown on silicon. The graphene grains show a clear contrast difference with the silicon surface, and most of the grains exhibit a regular, hexagon-derived shape, similar to the single-crystal graphene on $\mathrm{BN}$ or $\mathrm{Si}_{3} \mathrm{~N}_{4}$ substrates [27, 31]. The high-magnification AFM topography and phase image (Fig. 2c, d) also present a similar feature of a hexagon-derived shape, which further indicates the good crystallinity of graphene on silicon.

To better understand the nucleation and growth of graphene on single-crystal silicon, AFM images were recorded for a series of samples grown at temperatures between 900 and $930{ }^{\circ} \mathrm{C}$ (Fig. 3a-f). At the relatively low temperatures (900 and $905^{\circ} \mathrm{C}$ ), only few graphene domains appear (Fig. 3a, b). We can clearly distinguish two kinds of graphene domains, atomically flat mono- or bilayer graphene domains (lateral size of $\sim 130 \mathrm{~nm}$ ) and larger concave bilayer graphene domains $(\sim 160 \mathrm{~nm})$. These were confirmed by the height profiles shown at the bottom of Fig. 3a, b. At the more elevated temperature $\left(915^{\circ} \mathrm{C}\right)$, the number of graphene domains increases. This is accompanied by larger domain sizes as shown in Fig. 3c. Moreover, only a few core-bulging graphene domains (small convex spots on the center) emerge, which are marked by a red circle. The concave surface still dominates among the graphene grains.

After a further increase in the growth temperature, the core-bulging graphene domains gradually dominate in all AFM images. The height profiles, along the black lines in Fig. 3d-f, clearly show the formation of the core-bulging graphene domains, where the thickness changes from 1.9 to $2.9 \mathrm{~nm}$ (3-5 graphene layers). In fact, due to the extremely low roughness $(\sim 0.3 \mathrm{~nm})$ of the single-crystal silicon surface, three distinct areas are revealed in the AFM height images. These are highlighted by circles and arrows along the height profile lines. The light blue, blue, and red circles and arrows correspond to flat, concave, and bulging graphene domains, respectively. Moreover, even in samples dominated by the bulging domains, small flat monolayer or bilayer domains and concave bilayer domains also exist in the AFM image (light blue and blue circles and arrows in Fig. 3d-f). They show the roadmap of the domain evolution of initially small graphene grains. Figure $3 \mathrm{~g}$, h shows the corresponding AFM phase information at 900 and $930{ }^{\circ} \mathrm{C}$. Despite the height variation in the concave bilayer or core-bulging single domain, the AFM phase image reveals a component with a contrast different from that of silicon. This further confirms that the domains are composed of graphene. 
(a)
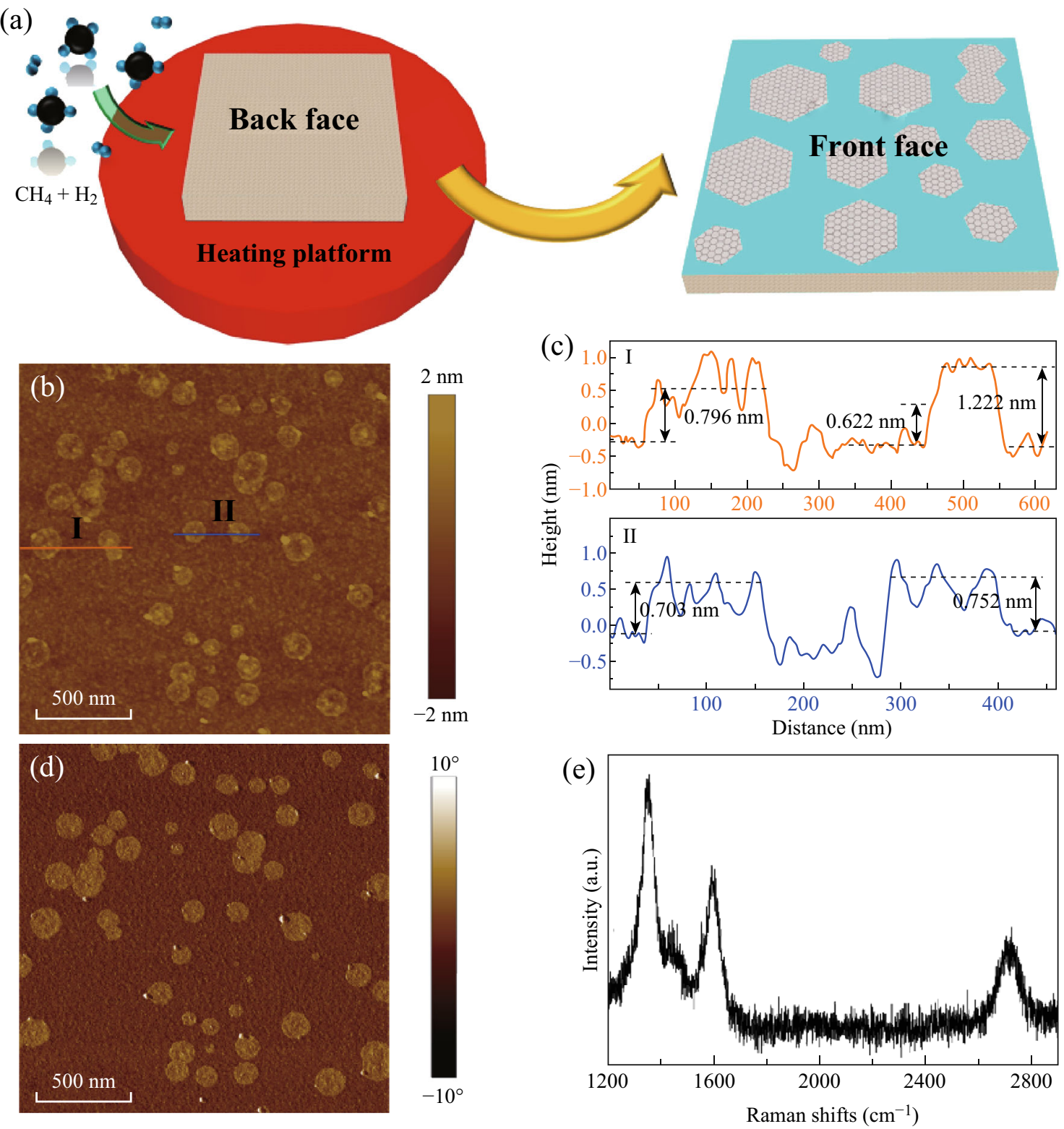

Fig. 1 Designed metal-free CVD synthesis and characterization of the as-grown graphene domains on silicon (100) substrates at $910{ }^{\circ} \mathrm{C}$. a Schematic diagram of the graphene domains grown on the silicon surface. The substrate was placed upside-down in order to capture more methane molecules during growth, which actually occurs on the front face. b Typical AFM height image. c Corresponding AFM height profile along the lines I, II in Fig. 1b. d Corresponding AFM phase image. e Raman spectra of graphene grown on silicon (100) substrates
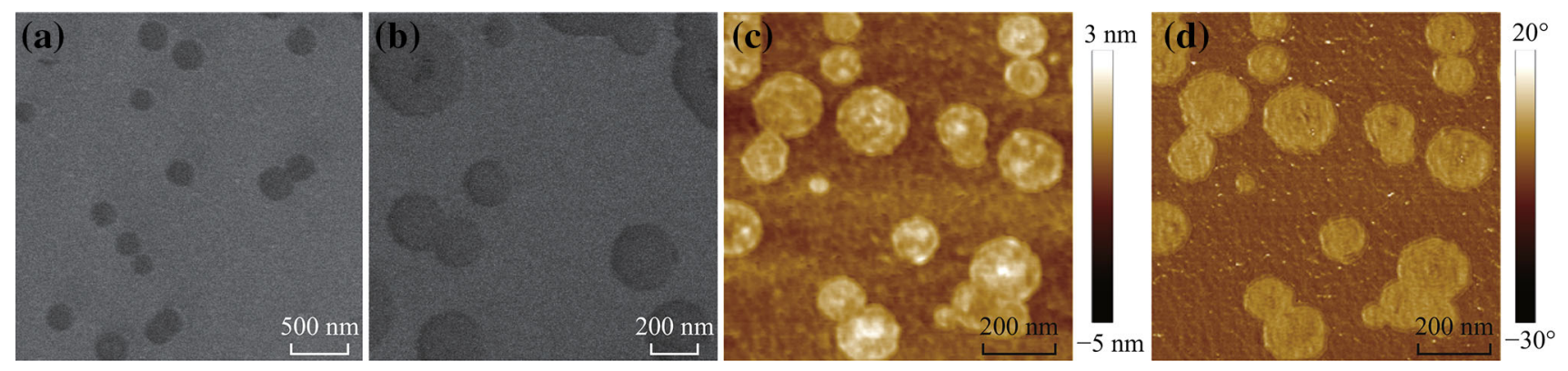

Fig. 2 a, b High-resolution SEM images of the graphene grains on silicon. $\mathbf{c}$ High-magnification AFM topography and $\mathbf{d}$ corresponding phase image of the graphene grains on silicon 

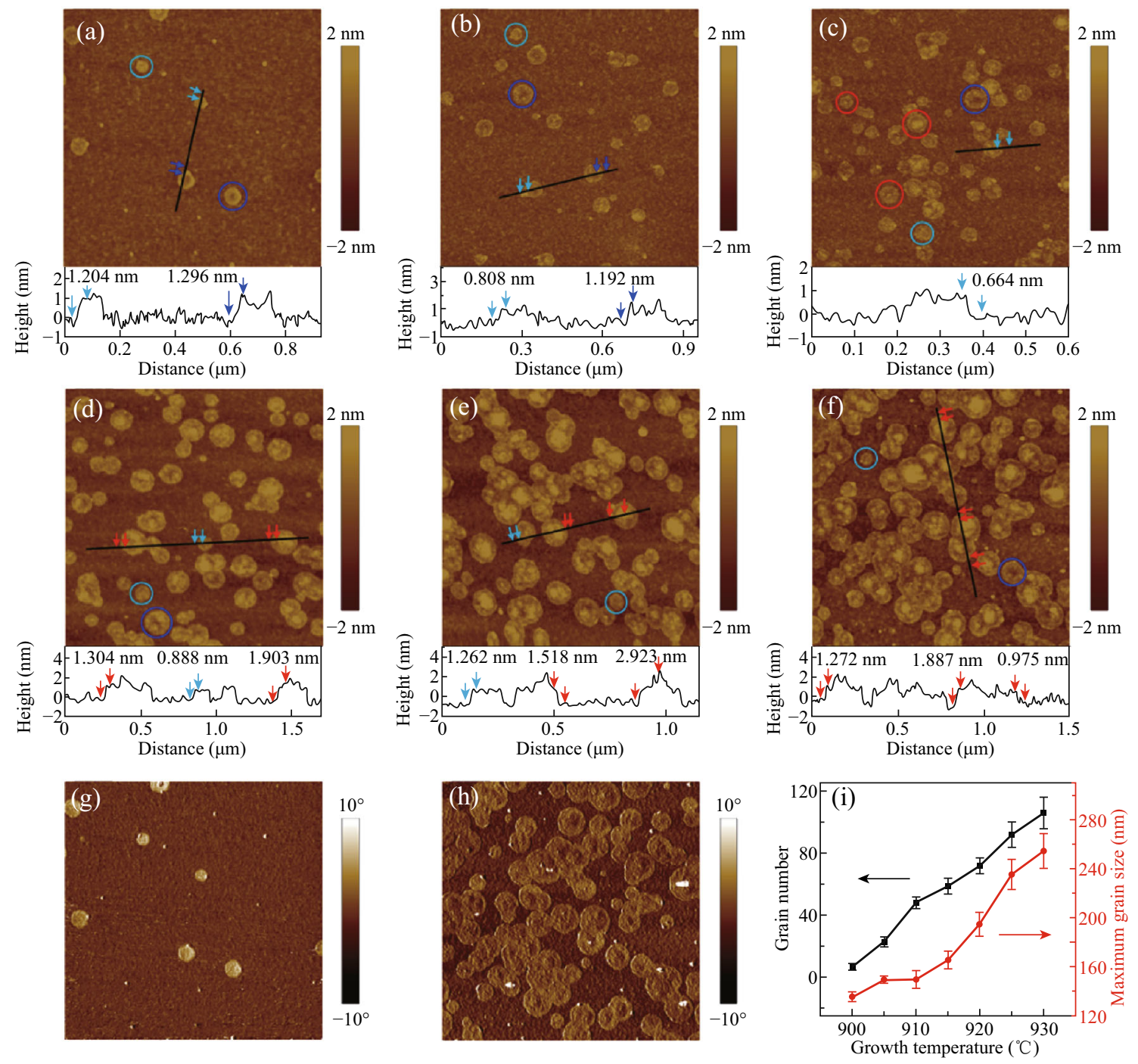

Fig. 3 Investigation of graphene nucleation and growth on silicon (100). a-f AFM height image of graphene domains directly grown on silicon (100) substrates for $1 \mathrm{~h}$ at the following temperatures: a $900{ }^{\circ} \mathrm{C}, \mathbf{b} 905{ }^{\circ} \mathrm{C}, \mathbf{c} 915{ }^{\circ} \mathrm{C}, \mathbf{d ~} 920{ }^{\circ} \mathrm{C}, \mathbf{e} 925{ }^{\circ} \mathrm{C}$, and f $930{ }^{\circ} \mathrm{C}$. The bottom row shows the corresponding height profile along the black line. The arrows indicate the height differences between the corresponding two points. Light blue, blue, and red circles correspond to flat, concave, and bulging graphene domains, respectively. The scan size is $2 \mu \mathrm{m}$. g, $\mathbf{h}$ The corresponding AFM phase images of graphene grown at 900 and $930{ }^{\circ} \mathrm{C}$, respectively. $\mathbf{i}$ The average grain number and the maximum grain size as a function of growth temperature

The average grain number and the maximum grain size vary as a function of growth temperature in Fig. 3i. Clearly, a higher growth temperature causes a larger domain size and higher nucleation density. This indicates that temperature is a key factor in controlling graphene nucleation and growth. Higher temperatures were also tried for the growth of graphene. However, we found that the surface of silicon was destroyed at temperatures higher than $950{ }^{\circ} \mathrm{C}$ (silicon reacts with the active hydrocarbon radicals, Fig. S3). On the other hand, there are other ways of growing larger graphene domains besides increasing the temperature. For example, it has been reported that trace oxygen can effectively accelerate graphene domain growth, for both metallic and dielectric substrates [18, 39]. A further study that explores trace oxygen-aided synthesis of large-area graphene layers is ongoing.

Spectroscopic characterizations were further conducted to probe the microstructural changes in graphene for different growth temperatures. Figure 4a shows the typical C $1 \mathrm{~s}$ spectrum for direct growth of graphene on a silicon (100) substrate at $910{ }^{\circ} \mathrm{C}$. This spectrum has been fitted with several peaks assigned to $s p^{2}$ carbon $(284.6 \mathrm{eV}), \mathrm{C}-\mathrm{H}$ $(285.1 \mathrm{eV})$, and a broad $\mathrm{C}-\mathrm{O}(286.4 \mathrm{eV})$ peak. The dominant peak of the $\mathrm{sp}^{2}$ feature confirms the presence of graphene. The $\mathrm{C}-\mathrm{H}\left(s p^{3}\right.$ carbon) signal should be due to the $\mathrm{H}$-terminated graphene edges, which is consistent with the Raman spectra in Fig. 1e. In the XPS Si $2 p$ spectra in Fig. $4 b$, a strong $\mathrm{Si}-\mathrm{O}$ peak and a weak $\mathrm{Si}-\mathrm{Si}$ peak appear 

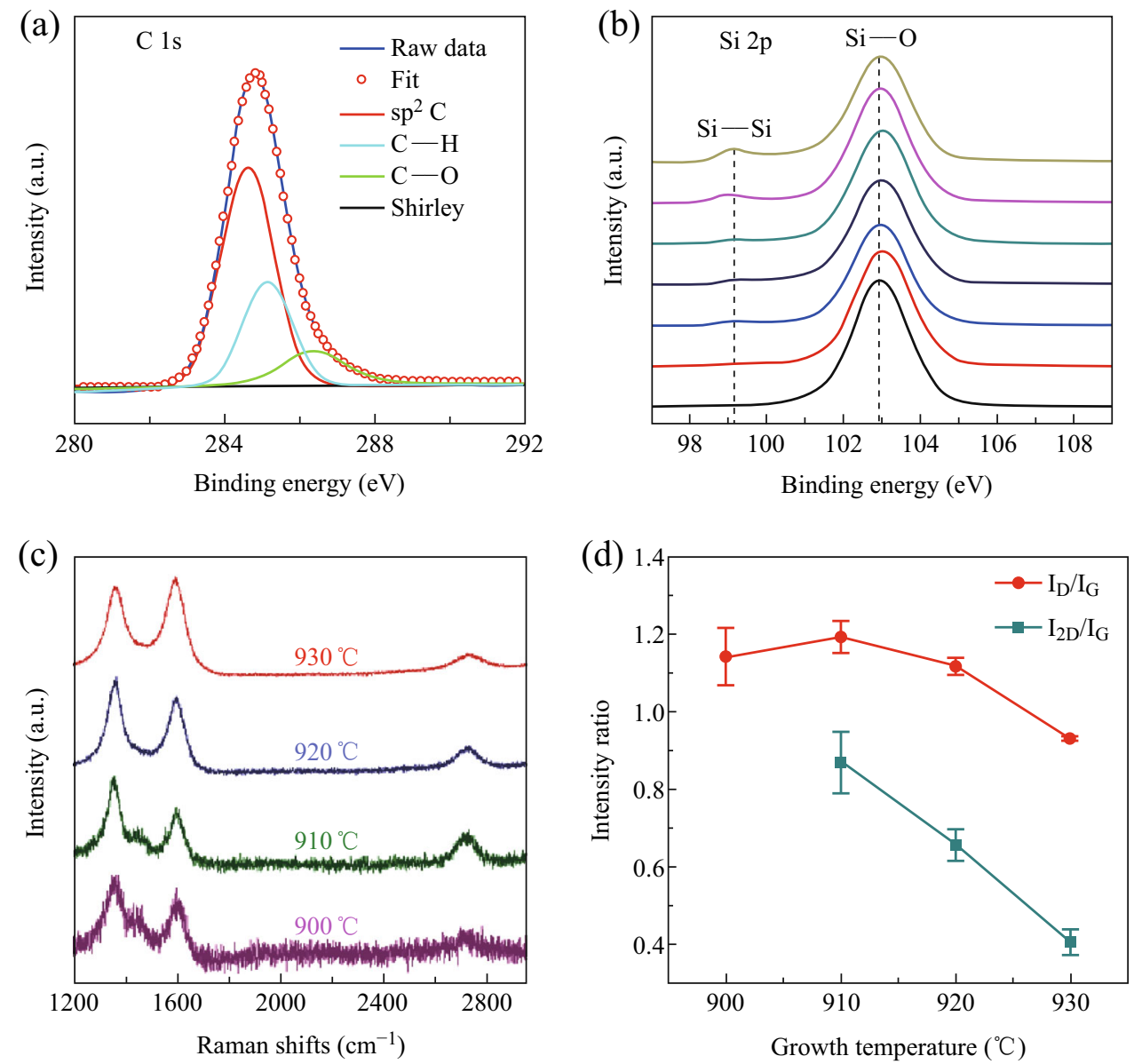

Fig. 4 Spectroscopic characteristics of graphene grown directly on silicon. a C 1 s XPS spectrum of as-grown graphene. b Si $2 p$ XPS spectra of graphene at various growth temperatures, from bottom to top are $900,905,910,915,920,925$, and $930{ }^{\circ} \mathrm{C}$, respectively. c Representative Raman spectra of graphene at various growth temperatures. d Ratio of the Raman D band intensity to that of the $\mathrm{G}$ band $\left(I_{\mathrm{D}} / I_{\mathrm{G}}\right)$, red, and the $2 \mathrm{D}$ band intensity to the $\mathrm{G}$ band $\left(I_{2 \mathrm{D}} / I_{\mathrm{G}}\right)$, light blue, as a function of growth temperature

at $\sim 103$ and $\sim 99 \mathrm{eV}$, respectively. The $\mathrm{Si}-\mathrm{O}$ peak is the result of the oxidization of silicon. The oxidization should have occurred only after the silicon substrate was exposed to air because the sample was annealed in a reducing atmosphere. We found that the intensity of the Si-Si peak increases with increasing growth temperature. This should be due to the increasing coverage of the graphene region because graphene prevents the oxidation of $\mathrm{Si}$ in air. The lack of the Si-C peak at approximately $100.3 \mathrm{eV}$ indicates that the interaction between the graphene and silicon substrate is weak, and no buffer layer of $\mathrm{Si}-\mathrm{C}$ was formed during the growth process (Fig. S4). Contrary to the observations of Hackley and Trung [32, 33], the lack of the $\mathrm{Si}-\mathrm{C}$ peak should be due to the following reason. In our CVD system, carbon is produced by the pyrolysis of methane. Without metallic catalysts, only a small number of $\mathrm{C}$ ions with weak mobility and activity were produced. Therefore, they would not react with silicon unless under much higher temperature, as demonstrated by Ki-Bum Kim et al. [34]. The temperature-dependent structure of graphene was further evaluated by Raman spectroscopy, as shown in Fig. 4c, d. Figure 4d shows the changes in the Raman characteristic signals for graphene (the intensity ratio of the $\mathrm{D}$ and $\mathrm{G}$ band, $I_{\mathrm{D}} / I_{\mathrm{G}}$, and the intensity ratio of the $2 \mathrm{D}$ band to the $\mathrm{G}$ band, $I_{2 \mathrm{D}} / I_{\mathrm{G}}$ ) with varying growth temperatures. The $I_{\mathrm{D}} / I_{\mathrm{G}}$ ratio is typically used to evaluate the quality of graphene. We found that the $I_{\mathrm{D}} / I_{\mathrm{G}}$ value increases slightly for graphene growth from 900 to $910{ }^{\circ} \mathrm{C}$. This can be due to the presence of more edges for the dominant concave bilayer graphene domains for samples grown at $910^{\circ} \mathrm{C}$. As the growth temperatures increase to 910 and $930{ }^{\circ} \mathrm{C}$, the enlarged graphene domains (Fig. 3i) lead to a gradual reduction in the $I_{\mathrm{D}} / I_{\mathrm{G}}$ value. The $2 \mathrm{D}$ band to $\mathrm{G}$ band ratio $\left(I_{2 \mathrm{D}} / I_{\mathrm{G}}\right)$ is commonly used as an indicator of the number of graphene layers $[40,41]$. The $I_{2 \mathrm{D}} / I_{\mathrm{G}}$ value decreases from 0.87 to 0.41 when the growth temperature increases from 910 to $930{ }^{\circ} \mathrm{C}$ (Raman mapping of $I_{2 \mathrm{D}} / I_{\mathrm{G}}$ for the sample grown at $905{ }^{\circ} \mathrm{C}$ is shown in Fig. S5). This 
suggests the layer propagation of graphene domains, which is consistent with our AFM observations.

Based on the above experiments, an integrated growth model considering the nucleation and growth process is proposed. In the first stage, due to the catalytically inert nature of silicon, the thermal self-decomposition of methane produces active carbon species that initiate graphene nucleation (Fig. 5a) [42]. Due to the upside-downplaced substrate, the super-saturation of carbon radicals and high collision frequency promotes the anchoring of carbon atoms onto the silicon surface and facilitates graphene nucleation. It was also reported that the high density of dangling bonds on a silicon surface could enhance nucleation [34, 43]. After the nucleation process, the growth of graphene follows the van der Waals growth model, and the silicon only acts as a support to define the growth plane. In fact, the van der Waals growth model has been successfully applied in growing graphene on insulators [26, 42] and other 2-D materials on silicon [44]. The active carbon decomposed from the continuously supplied methane results in the subsequent growth of graphene
(Fig. 5b). The in-plane propagation of graphene initially occurs with carbon atoms landing on the edge of a stable nucleus and forming bonds with the carbon atoms on the edge of the domains. This produces small, flat monolayer, or bilayer, graphene domains. For the monolayer graphene domain, due to the weak interaction between silicon and graphene and the large number of dangling carbon atoms existing at the edge of the domain, the inplane propagation may be blocked by the formation of $\mathrm{C}-\mathrm{H}$ bands, which are indicated by the XPS results shown in Fig. 4a [45]. As a result, in-plane propagation stops and the graphene domain reach a critical stable size $(\sim 130 \mathrm{~nm})$.

At a higher temperature, both the hydrocarbon radicals and the silicon surface should become more active, and an additional layer of graphene forms on the active edge of the monolayer graphene domain. The growth mechanism of graphene changes to edge-propagation instead. Therefore, concave bilayer domains with larger size are formed, as depicted in Fig. 5c. The small bilayer graphene domains, however, do not participate in the edge-propagation process (light blue circle in Fig. 3a, e). This is mainly due to
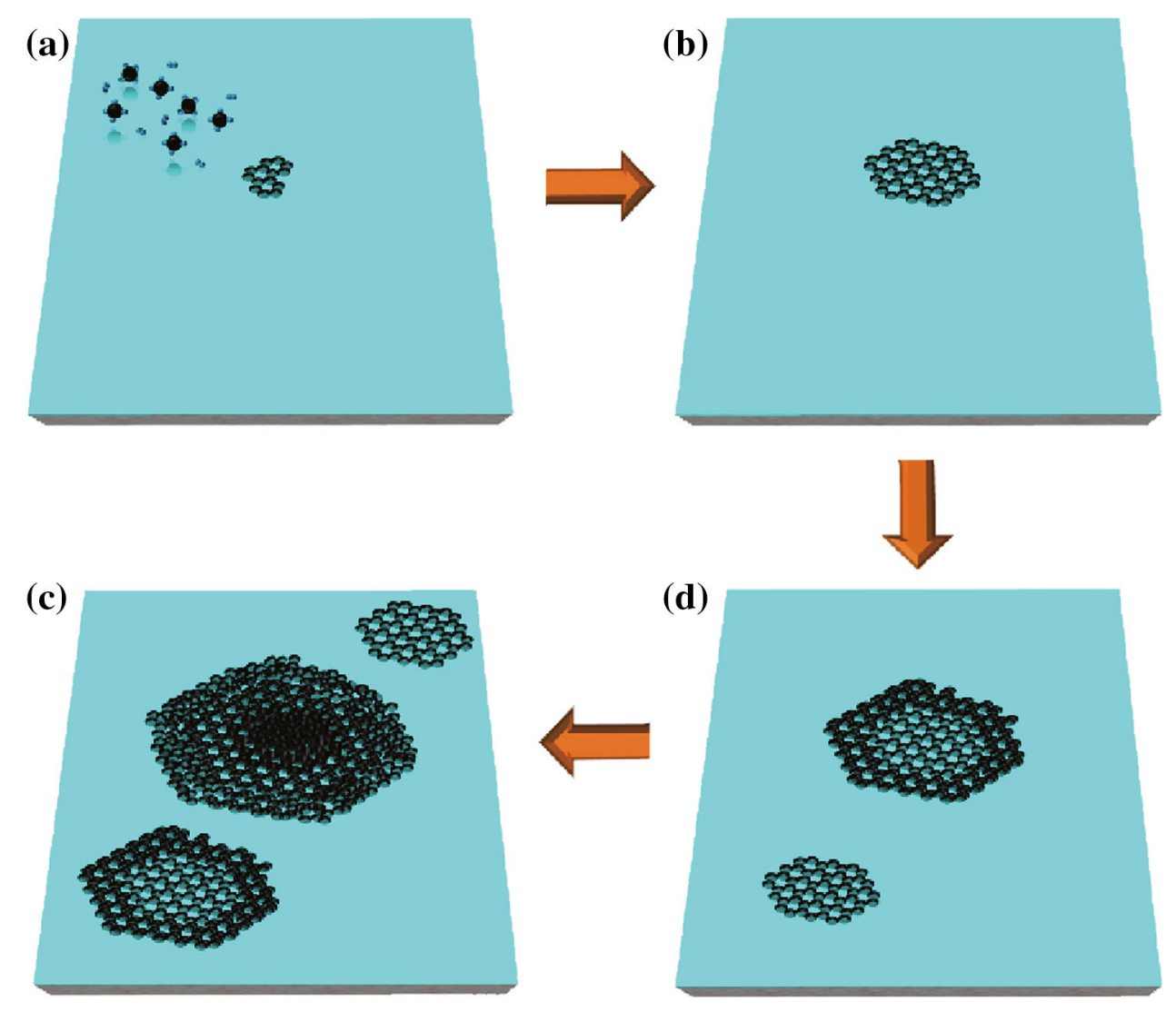

Fig. 5 Schematic of the direct nucleation and growth process of graphene domains on silicon substrates using metal-free APCVD. a Methane decomposition and super-saturated, active carbon species anchored on the silicon surface. This facilitates graphene nucleation. $\mathbf{b}$ In-plane propagation with carbon atoms landing at the edge of stable nuclei and the formation of small flat monolayer or bilayer graphene domains. c Edge-propagation process for bilayer graphene depositions at the active edge of the monolayer graphene domains, and the formation of larger, concave bilayer graphene domains. d Core-propagation process with more graphene layers nucleating in the core of the concave graphene domains, and stabilization of the bigger bulging graphene domains at higher growth temperatures 
the strong layer interaction in the bilayer and the obtuse nature of the bilayer graphene domain edge [46, 47], which is also confirmed by the stable boundary of the concave bilayer domains. With further increase in growth temperature, the reaction is more active, thus accelerating the propagation process and producing larger graphene domains. Furthermore, superfluous active carbons begin to nucleate in the core of already-grown, concave bilayer graphene domains. The core-propagation process occurs and generates bulging, few-layer graphene domains (Fig. 5d) [30].

The growth of graphene was further explored using different orientations and doping types of single-crystal silicon substrates (including the [100], [111], and [110] surface, and $n$-type and $p$-type silicon). We found that the growth regimes of graphene are independent of the $\mathrm{Si}$ surface structure, further indicating that the direct growth of graphene on silicon is a self-propagating process and follows the van der Waals growth model. The growth of graphene on $\mathrm{Si}$ is mainly controlled by the temperature and the confined, local environment of the precursors.

\section{Conclusion}

In summary, through the optimization of the growth conditions, we demonstrate the direct growth of graphene onto a single-crystal silicon surface using metal-free APCVD, which is confirmed by AFM, SEM, Raman, and XPS characterizations. In addition, in-plane propagation, edgepropagation, and core-propagation processes were proposed to evaluate the sequentially changing graphene domains. A better understanding of the direct nucleation and growth mechanisms may enable the synthesis of largearea and layer-controlled, high-quality graphene on singlecrystal silicon substrates.

Acknowledgements The authors thank Lijuan Zhang for the help of AFM characterizations and Yi Gao for the helpful discussion. This work was financially supported by the National Natural Science Foundation of China (NSFC) (Grant Nos. 11405253, 11225527, 11575283, 11205235, U1632129, U1332205), Shanghai Science Foundation (14YF1407500), and the Youth Innovation Promotion Association CAS (2016237). The authors thank the staff at the beamlines BL14B1 and BL08U of the Shanghai Synchrotron Radiation Facility.

Open Access This article is distributed under the terms of the Creative Commons Attribution 4.0 International License (http://crea tivecommons.org/licenses/by/4.0/), which permits unrestricted use, distribution, and reproduction in any medium, provided you give appropriate credit to the original author(s) and the source, provide a link to the Creative Commons license, and indicate if changes were made.

\section{References}

1. A.K. Geim, K.S. Novoselov, The rise of grapheme. Nat. Mater. 6(3), 183-191 (2007). https://doi.org/10.1038/nmat1849

2. K.S. Novoselov, A.K. Geim, S.V. Morozov, D. Jiang, Y. Zhang, S.V. Dubonos, I.V. Grigorieva, A.A. Firsov, Electric field effect in atomically thin carbon films. Science 306(5696), 666-669 (2004). https://doi.org/10.1126/science.1102896

3. X. Li, W. Cai, L. Colombo, R.S. Ruoff, Evolution of graphene growth on $\mathrm{Ni}$ and $\mathrm{Cu}$ by carbon isotope labeling. Nano Lett. 9(12), 4268-4272 (2009). https://doi.org/10.1021/n1902515k

4. K.S. Kim, Y. Zhao, H. Jang, S.Y. Lee, J.M. Kim, J.-H. Ahn, P. Kim, J.-Y. Choi, B.H. Hong, Large-scale pattern growth of graphene films for stretchable transparent electrodes. Nature 457(7230), 706-710 (2009). https://doi.org/10.1038/nature07719

5. L. Gao, W. Ren, H. Xu, L. Jin, Z. Wang et al., Repeated growth and bubbling transfer of graphene with millimetre-size singlecrystal grains using platinum. Nat. Commun. 3, 699 (2012). https://doi.org/10.1038/ncomms1702

6. Y. Liu, M. Xu, X. Zhu, M. Xie, Y. Su, N. Hu, Z. Yang, Y.F. Zhang, Synthesis of carbon nanotubes on graphene quantum dot surface by catalyst free chemical vapor deposition. Carbon $\mathbf{6 8}$, 399-405 (2014). https://doi.org/10.1016/j.carbon.2013.11.016

7. X. Li, W. Cai, J. An, S. Kim, J. Nah et al., Large-area synthesis of high-quality and uniform graphene films on copper foils. Science 324(5932), 1312-1314 (2009). https://doi.org/10.1126/science. 1171245

8. Y. Jia, X. Gong, P. Peng, Z. Wang, Z. Tian, L. Ren, Y. Fu, H. Zhang, Toward high carrier mobility and low contact resistance: laser cleaning of PMMA residues on graphene surfaces. Nano Micro Lett. 8(4), 336-346 (2016). https://doi.org/10.1007/ s40820-016-0093-5

9. J.-H. Lee, E.K. Lee, W.-J. Joo, Y. Jang, B.-S. Kim et al., Waferscale growth of single-crystal monolayer graphene on reusable hydrogen-terminated germanium. Science 344(6181), 286-289 (2014). https://doi.org/10.1126/science. 1252268

10. A.M. Scaparro, V. Miseikis, C. Coletti, A. Notargiacomo, M. Pea, M. De Seta, L. Di Gaspare, Investigating the CVD synthesis of graphene on Ge (100): toward layer-by-layer growth. ACS Appl. Mater. Interfaces 8(48), 33083-33090 (2016). https://doi.org/10. 1021/acsami.6b11701

11. S. Tongay, T. Schumann, X. Miao, B.R. Appleton, A.F. Hebard, Tuning Schottky diodes at the many-layer-graphene/semiconductor interface by doping. Carbon 49(6), 2033-2038 (2011). https://doi.org/10.1016/j.carbon.2011.01.029

12. S. Tongay, M. Lemaitre, X. Miao, B. Gila, B.R. Appleton, A.F. Hebard, Rectification at graphene-semiconductor interfaces: zero-gap semiconductor-based diodes. Phys. Rev. X 2(1), 011002 (2012). https://doi.org/10.1103/PhysRevX.2.011002

13. Y. Lin, X. Li, D. Xie, T. Feng, Y. Chen et al., Graphene/semiconductor heterojunction solar cells with modulated antireflection and graphene work function. Energy Environ. Sci. 6(1), 108-115 (2013). https://doi.org/10.1039/C2EE23538B

14. C.-C. Chen, M. Aykol, C.-C. Chang, A.F.J. Levi, S.B. Cronin, Graphene-silicon Schottky diodes. Nano Lett. 11(5), 1863-1867 (2011). https://doi.org/10.1021/nl104364c

15. X. An, F. Liu, Y.J. Jung, S. Kar, Tunable graphene-silicon heterojunctions for ultrasensitive photodetection. Nano Lett. 13(3), 909-916 (2013). https://doi.org/10.1021/nl303682j

16. K. Ruan, K. Ding, Y. Wang, S. Diao, Z. Shao, X. Zhang, J. Jie, Flexible graphene/silicon heterojunction solar cells. J. Mater. Chem. A 3(27), 14370-14377 (2015). https://doi.org/10.1039/ C5TA03652F

17. W. Strupinski, K. Grodecki, A. Wysmolek, R. Stepniewski, T. Szkopek et al., Graphene epitaxy by chemical vapor deposition 
on SiC. Nano Lett. 11(4), 1786-1791 (2011). https://doi.org/10. 1021/nl200390e

18. J. Chen, Y. Wen, Y. Guo, B. Wu, L. Huang et al., Oxygen-aided synthesis of polycrystalline graphene on silicon dioxide substrates. J. Am. Chem. Soc. 133(44), 17548-17551 (2011). https:// doi.org/10.1021/ja2063633

19. D. Wei, Y. Lu, C. Han, T. Niu, W. Chen, A.T.S. Wee, Critical crystal growth of graphene on dielectric substrates at low temperature for electronic devices. Angew. Chem. Int. Ed. 52(52), 14121-14126 (2013). https://doi.org/10.1002/anie.201306086

20. Q. Wang, P. Zhang, Q. Zhuo, X. Lv, J. Wang, X. Sun, Direct synthesis of Co-doped graphene on dielectric substrates using solid carbon sources. Nano Micro Lett. 7(4), 368-373 (2015). https://doi.org/10.1007/s40820-015-0052-6

21. Q.-Q. Zhuo, Q. Wang, Y.-P. Zhang, D. Zhang, Q.-L. Li et al., Transfer-free synthesis of doped and patterned graphene films. ACS Nano 9(1), 594-601 (2015). https://doi.org/10.1021/ nn505913v

22. W.T.E. Beld, A. Berga, J.C.T. Eijkela, Spatial control of direct chemical vapor deposition of graphene on silicon dioxide by directional copper dewetting. RSC Adv. 6(92), 89380-89386 (2016). https://doi.org/10.1039/C6RA16935J

23. J. Sun, Y. Chen, M.K. Priydarshi, Z. Chen, A. Bachmatiuk et al., Direct chemical vapor deposition-derived graphene glasses targeting wide ranged applications. Nano Lett. 15(9), 5846-5854 (2015). https://doi.org/10.1021/acs.nanolett.5b01936

24. A. Ismach, C. Druzgalski, S. Penwell, A. Schwartzberg, M. Zheng, A. Javey, J. Bokor, Y. Zhang, Direct chemical vapor deposition of graphene on dielectric surfaces. Nano Lett. 10(5), 1542-1548 (2010). https://doi.org/10.1021/n19037714

25. M.A. Fanton, J.A. Robinson, C. Puls, Y. Liu, M.J. Hollander et al., Characterization of graphene films and transistors grown on sapphire by metal-free chemical vapor deposition. ACS Nano 5(10), 8062-8069 (2011). https://doi.org/10.1021/nn202643t

26. J. Hwang, M. Kim, D. Campbell, H.A. Alsalman, J.Y. Kwak et al., Van der waals epitaxial growth of graphene on sapphire by chemical vapor deposition without a metal catalyst. ACS Nano 7(1), 385-395 (2012). https://doi.org/10.1021/nn305486x

27. J. Chen, Y. Guo, Y. Wen, L. Huang, Y. Xue et al., Two-stage metal-catalyst-free growth of high-quality polycrystalline graphene films on silicon nitride substrates. Adv. Mater. 25(7), 992-997 (2013). https://doi.org/10.1002/adma.201202973

28. J. Sun, T. Gao, X. Song, Y. Zhao, Y. Lin et al., Direct growth of high-quality graphene on high- $\kappa$ dielectric $\mathrm{SrTiO}_{3}$ substrates. J. Am. Chem. Soc. 136(18), 6574-6577 (2014). https://doi.org/ $10.1021 / \mathrm{ja} 5022602$

29. M. Wang, S.K. Jang, W.J. Jang, M. Kim, S.Y. Park et al., A platform for large-scale graphene electronics-CVD growth of single-layer graphene on CVD-grown hexagonal boron nitride. Adv. Mater. 25(19), 2746-2752 (2013). https://doi.org/10.1002/ adma.201204904

30. S. Tang, G. Ding, X. Xie, J. Chen, C. Wang, X. Ding, F. Huang, W. Lu, M. Jiang, Nucleation and growth of single crystal graphene on hexagonal boron nitride. Carbon 50(1), 329-331 (2012). https://doi.org/10.1016/j.carbon.2011.07.062

31. S. Tang, H. Wang, H.S. Wang, Q. Sun, X. Zhang et al., Silanecatalysed fast growth of large single-crystalline graphene on hexagonal boron nitride. Nat. Commun. 6, 6499 (2015). https:// doi.org/10.1038/ncomms 7499
32. J. Hackley, D. Ali, J. DiPasquale, J. Demaree, C. Richardson, Graphitic carbon growth on $\mathrm{Si}(111)$ using solid source molecular beam epitaxy. Appl. Phys. Lett. 95(13), 133114 (2009). https:// doi.org/10.1063/1.3242029

33. P.T. Trung, F. Joucken, J. Campos-Delgado, J.-P. Raskin, B. Hackens, R. Sporken, Direct growth of graphitic carbon on Si(111). Appl. Phys. Lett. 102(1), 013118 (2013). https://doi.org/ 10.1063/1.4773989

34. K.B. Kim, C.M. Lee, J. Choi, Catalyst-free direct growth of triangular nano-graphene on all substrates. J. Phys. Chem. C 115(30), 14488-14493 (2011). https://doi.org/10.1021/jp2017709

35. D. Zhu, H. Gao, X. Zhang, T. Yang, L. Li et al., Real-time observation of graphene layer growth: coupling of the interlayer spacing with thickness. Carbon 94, 775-780 (2015). https://doi. org/10.1016/j.carbon.2015.07.047

36. H. Wang, X. Xu, J. Li, L. Lin, L. Sun et al., Surface monocrystallization of copper foil for fast growth of large singlecrystal graphene under free molecular flow. Adv. Mater. 28(40), 8968-8974 (2016). https://doi.org/10.1002/adma.201603579

37. G. Socrates, Infrared and Raman Characteristic Group Frequencies: Tables and Charts (Wiley, Chichester, 2004)

38. A.C. Ferrari, J. Robertson, Resonant Raman spectroscopy of disordered, amorphous, and diamond like carbon. Phys. Rev. B 64(7), 075414 (2001). https://doi.org/10.1103/PhysRevB.64. 075414

39. Y. Hao, M.S. Bharathi, L. Wang, Y. Liu, H. Chen et al., The role of surface oxygen in the growth of large single-crystal graphene on copper. Science 342(6159), 720-723 (2013). https://doi.org/ $10.1126 /$ science. 1243879

40. A.C. Ferrari, J.C. Meyer, V. Scardaci, C. Casiraghi, M. Lazzeri et al., Raman spectrum of graphene and graphene layers. Phys. Rev. Lett. 97(18), 187401 (2006). https://doi.org/10.1103/Phys RevLett.97.187401

41. J. Lee, K.S. Novoselov, H.S. Shin, Interaction between metal and graphene: dependence on the layer number of graphene. ACS Nano 5(1), 608-612 (2011). https://doi.org/10.1021/nn103004c

42. G. Lippert, J. Dabrowski, M. Lemme, C. Marcus, O. Seifarth, G. Lupina, Direct graphene growth on insulator. Phys. Status Solidi B 248(11), 2619-2622 (2011). https://doi.org/10.1002/pssb. 201100052

43. G. Hong, Q.H. Wu, J. Ren, S.T. Lee, Mechanism of non-metal catalytic growth of graphene on silicon. Appl. Phys. Lett. 100(23), 231604 (2012). https://doi.org/10.1063/1.4726114

44. L. Huang, Y. Yu, C. Li, L. Cao, Substrate mediation in vapor deposition growth of layered chalcogenide nanoplates: a case study of $\mathrm{SnSe}_{2}$. J. Phys. Chem. C 117(12), 6469-6475 (2013). https://doi.org/10.1021/jp400274a

45. M. Losurdo, M.M. Giangregorio, P. Capezzuto, G. Bruno, Graphene CVD growth on copper and nickel: role of hydrogen in kinetics and structure. Phys. Chem. Chem. Phys. 13(46), 20836-20843 (2011). https://doi.org/10.1039/c1cp22347j

46. L. Qi, J.Y. Huang, J. Feng, J. Li, In situ observations of the nucleation and growth of atomically sharp graphene bilayer edges. Carbon 48(8), 2354-2360 (2010). https://doi.org/10.1016/ j.carbon.2010.03.018

47. J.Y. Huang, F. Ding, B.I. Yakobson, P. Lu, L. Qi, J. Li, In situ observation of graphene sublimation and multi-layer edge reconstructions. Proc. Natl. Acad. Sci. 106(25), 10103-10108 (2009). https://doi.org/10.1073/pnas.0905193106 